\title{
Consecuencias clínicas posgastrectomía vertical tubular: enfermedad por reflujo gastroesofágico
}

\author{
Italo Braghetto M. ${ }^{1}$
}

'Departamento de Cirugía Hospital Clínico Universidad

de Chile. Santiago, Chile.

Recibido el 5 de marzo

de 2018, aceptado para publicación el 7 de mayo de

Correspondencia a: Dr Italo Braghetto M. ibraghet@hcuch.c

\section{Clinical consequences post tubular vertical gastrectomy: gastroesophageal reflux disease}

Several publications report an increase in the rate of gastroesophageal reflux disease (GERD) after surgery, although there is still no consensus on this. The aim of this article is to show our own experience and literature regarding the clinical aspects of GERD disease after tubular vertical gastrectomy. From the analysis of our results and data from the literature, studies evaluating the prevalence of postoperative gastro-oesophageal reflux disease show that GVT can cause symptoms of de novo GERD or worsening of pre-existing GERD.

Key words: tubular vertical gastrectomy; gastroesophageal reflux disease; sequels.

\section{Resumen}

Varias publicaciones informan un aumento en la tasa de enfermedad por reflujo gastroesofágico (ERGE) después de la gastrectomía vertical tubular (GVT) o en manga a pesar que no existe aún consenso al respecto. El objetivo de este artículo es mostrar nuestra propia experiencia y de la literatura respecto a los aspectos clínicos de la enfermedad por ERGE después de la gastrectomía vertical tubular. Del análisis de nuestros resultados y de los datos de la literatura, los estudios que evalúan la prevalencia de la enfermedad de reflujo gastroesofágico posoperatorio muestran que la GVT puede provocar síntomas de ERGE de novo o empeoramiento de la ERGE preexistente.

Palabras clave: gastrectomía vertical tubular; reflujo gastroesofágico; secuelas.

\section{Introducción}

La gastrectomía vertical tubular (GVT) ha mostrado buenos resultados con respecto a la reducción de peso y la mejora en las comorbilidades, pero, varios estudios de seguimiento informan un aumento en la tasa de enfermedad por reflujo gastroesofágico (ERGE) después de la cirugía. La ERGE se describe como de novo o por agravamiento de los síntomas preexistentes. En la literatura no hay consenso sobre este tema, con varias publicaciones que muestran reducción en los síntomas de reflujo y muchos otros que muestran un aumento significativo en los síntomas de reflujo con o sin esofagitis ${ }^{1-7}$. El objetivo de este artículo es mostrar nuestra propia experiencia y lo conocido de la literatura respecto a los aspectos clínicos de la enfermedad por ERGE después de la gastrectomía vertical tubular.

\section{Consecuencias clínicas}

Como consecuencia de los factores comentados, la ERGE se observa con frecuencia después de la GVT. Los datos muestran un alto porcentaje de síntomas preoperatorios persistentes que ocurren en hasta el $84 \%^{2-7}$. Además, hay evidencias clínicas de aparición de novo de síntomas de ERGE, esofagitis y esófago de Barrett después de la gastrectomía en manga (rango 7,8 a 21\%). El 80\% de los pacientes sometidos a GVT tuvo un reflujo leve a severo, asociado con EEI incompetente u otros mecanismos fisiopatológicos $2,6,8-21$.

Para Tutuian ${ }^{18}$, la presencia de ERGP podría representar una contraindicación relativa para la GVT porque los pacientes pueden desarrollar ERGE y esofagitis después de ella. De acuerdo con esta idea, siempre excluimos a los pacientes con síntomas de 
reflujo o esofagitis endoscópica como candidatos para la GVT, prefiriendo en esos casos el bypass gástrico en Y de Roux como operación primaria.

\section{Síntomas}

La aparición de síntomas de ERGE y esofagitis es muy variable $2,6,11-27$. Incluyendo los datos más recientes sobre este punto, la mayoría de los estudios informaron un aumento en los síntomas de ERGE. La Tabla 4 describe los datos reportados por la literatura internacional. La mayoría de las publicaciones han mostrado síntomas después de la cirugía ${ }^{11-18,20-25}$. Gen$\mathrm{co}^{13}$ también demostró una mayor incidencia de síntomas de ERGE ( $42 \%$ de sus pacientes) en un promedio de 58 meses de seguimiento, y nosotros hemos observado esofagitis 9 años después de la operación. Tai ${ }^{14}$ demostró un aumento significativo en la prevalencia y severidad de los síntomas de ERGE de 2,1 a $47 \%$. Otras publicaciones sugieren una mejoría de los síntomas de reflujo después de la GVT. Albanopoulos ${ }^{27}$ informó la reducción del reflujo gastroesofágico en el $9,2 \%$, pero en el $11,4 \%$ de los casos, nuevos casos de ERGE aparecieron a los 36 meses. Goldenshluger ${ }^{28}$ informó recientemente una remisión del $60,7 \%$ para el reflujo gastroesofágico. Angrisani ${ }^{29}$ asocia la remisión de los síntomas de ERGE con el \% de pérdida de peso después de 5 años de seguimiento. Sin embargo, estos 3 últimos estudios no mencionaron los hallazgos endoscópicos después de la cirugía. La falta de correlación con otras evaluaciones objetivas como el examen endoscópico, la manometría o la prueba de reflujo ácido es un gran problema, responsable de la enorme variación de la tasa de síntomas de ERGE después de la GVT y muchos de los estudios presentan esta limitación (Tabla 1).

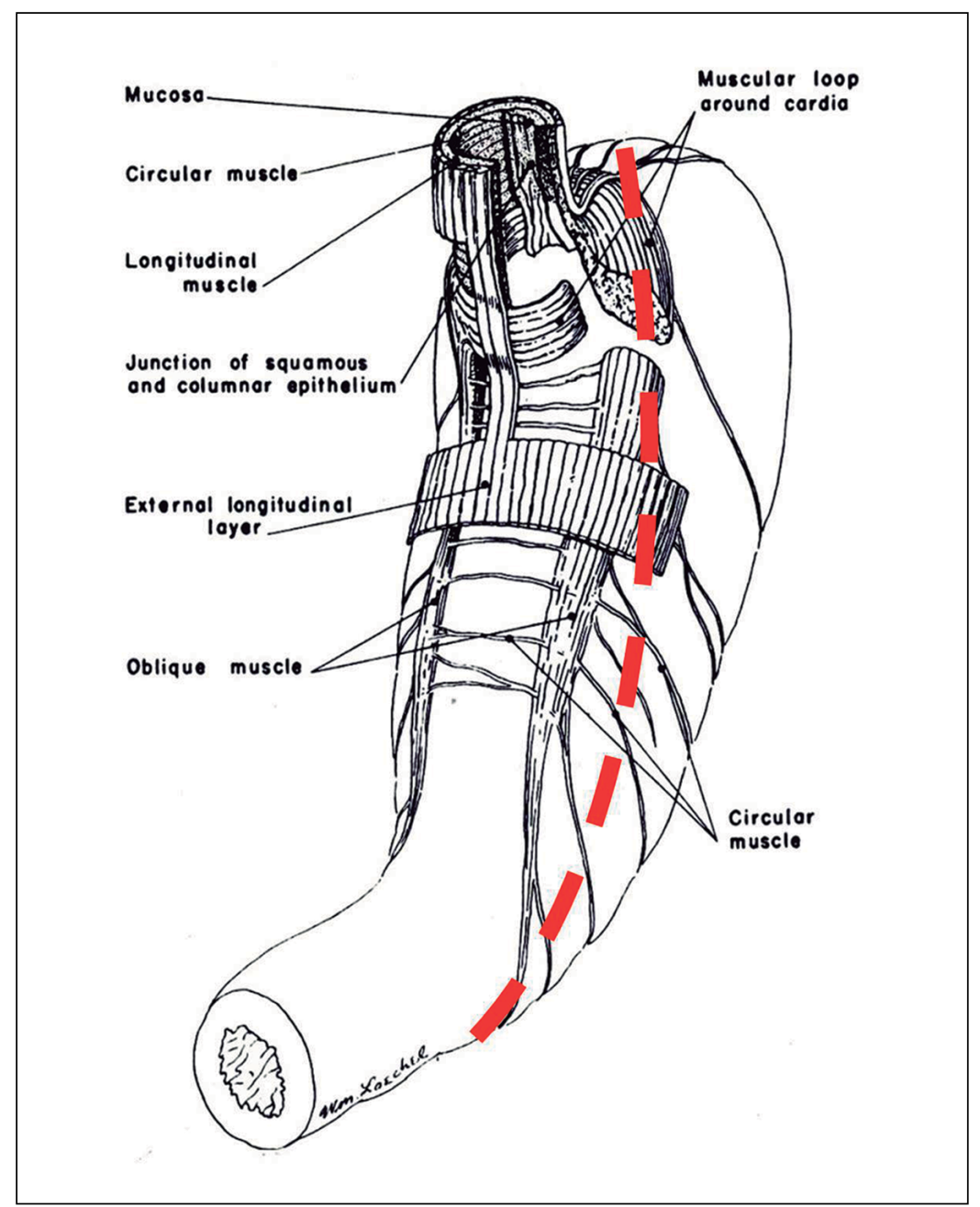

Figura 1. Línea de sección de fibras componentes del Esfínter Esofágico inferior durante la gastrectomía vertical tubular (Braghetto, Korn. IFSO , Montreal 2014).

Tabla 1. Síntomas de reflujo gastroesofágico posgastrectomía vertical tubular

\begin{tabular}{|c|c|c|c|c|c|}
\hline & n & Seguimiento & Síntomas & Promedio & (Rango) \\
\hline $\begin{array}{l}\text { Mejoría } \\
\text { Autores }(n=17)\end{array}$ & 3.289 & $1-5$ años & $\begin{array}{l}\text { Preoperatorios } \\
\text { Posoperatorios } \\
\% \text { mejoría } \\
\text { Síntomas de novo }\end{array}$ & $\begin{array}{l}27,3 \% \\
11,7 \% \\
46,3 \% \\
7,9 \%\end{array}$ & $\begin{array}{l}(3-49) \\
(3,1-27)^{*} \\
(24,5-95) \\
(3,2-17,7)\end{array}$ \\
\hline $\begin{array}{l}\text { Empeoramiento } \\
\text { Autores }(n=28)\end{array}$ & 10.810 & 1-11 años & $\begin{array}{l}\text { Preoperatorio } \\
\text { Posoperatorio } \\
\% \text { empeoramiento } \\
\text { Síntomas de novo }\end{array}$ & $\begin{array}{l}29,2 \% \\
38,6 \% \\
61,8 \% \\
24,5 \%\end{array}$ & $\begin{array}{l}(0-70) \\
(9-84) \\
(20-125) \\
(2,1-50)\end{array}$ \\
\hline \multicolumn{3}{|c|}{ Experiencia personal (657 paciente operados) $\rightarrow$} & $\begin{array}{l}313 \text { controlados } \\
\text { Preoperatorio } \\
\text { Posoperatorio }\end{array}$ & \multicolumn{2}{|c|}{$\begin{array}{l}\text { (5-9 años) } \\
0 \\
64,2 \% \text { (“de novo”) }\end{array}$} \\
\hline
\end{tabular}

\footnotetext{
$* 2$ autores $=$ hernioplastia hiatal concomitante.
} 


\section{Necesidad de tratamiento con inhibidores de la bomba de protones (IBP)}

Entre nuestros pacientes sometidos a GVT, 179 de ellos $(57,2 \%)$ han sido medicados con IBP debido a síntomas de reflujo. Esto está en concordancia con otras experiencias ${ }^{30-34}$. Catheline ${ }^{30}$, después de 5 años de seguimiento, informó un aumento del $200 \%$ en pacientes que requieren tratamiento con IBP, Carter $^{31}$ describió que el $33,8 \%$ de sus pacientes necesitan este tratamiento. En la experiencia de Kowalewski ${ }^{23}$, el $44 \%$ de los pacientes fueron tratados y Sheppard encontró un aumento significativo en el uso de IBP en pacientes con GVT $^{22}$. También en el artículo publicado recientemente por Mendeville ${ }^{24}$ se demostró aumento significativo en los síntomas de reflujo y uso de IBP en el $52 \%$ de los pacientes. Genco ${ }^{13}$ demostró que el $42 \%$ de los pacientes tenían una ingesta de IBP significativamente mayor en comparación con el preoperatorio. Zhang ${ }^{35}$ no publicó cambios significativos entre el tratamiento pre y posoperatorio. Muy pocos autores han informado disminución en el requerimiento de estos medicamentos después de GVT. Rebecchi ${ }^{36} \mathrm{a}$ los 2 años de seguimiento publicó una disminución del tratamiento con IBP en el $71 \%$ de los pacientes y Barry $^{37}$ encontró reducciones absolutas significativas en el uso de agentes antirreflujo; pero, en pacientes con síntomas preoperatorios, en solo unos pocos de ellos se redujo la medicación, lo que significa que los síntomas continuaron igualmente sin mejoría. En estas experiencias, no se menciona la evaluación endoscópica para excluir o confirmar la esofagitis después de la cirugía. En la experiencia reportada por Albanopoulos ${ }^{27}$, a los 3 años de seguimiento, el $33,4 \%$ necesita medicación después de GVT (Tabla 2).

\section{Esofagitis posoperatoria, esófago de Barrett y hernia hiatal después de GVT}

En una revisión de varios autores ${ }^{16,38-41}$, la aparición de esofagitis varía del $6,3 \%$ al $66,7 \%$. Tai ${ }^{14}$ publicó una prevalencia de esofagitis erosiva de 16,7 a $66,7 \%$, asociado con una mayor gravedad de los síntomas de ERGE relacionados con la presencia de una hernia hiatal después de la operación (27\%). En una publicación anterior ${ }^{42}$, encontramos esofagitis en $15,5 \%$ y se detectó esófago de Barrett en 1,2\% a los 3 años de seguimiento. En la actualidad, a los 5-9 años de seguimiento, el esófago de Barrett aumenta a 4,8\%. Muchos autores de los ya antes citados han señalado lo mismo ${ }^{12-17,20-28}$. De acuerdo con estas experiencias, hemos observado esofagitis endoscópica muy severa (Grado C) después de la GVT (Tabla 3).

Chuffart ${ }^{26}$ encontró $22 \%$ de los síntomas de ERGE de novo. Rengo ${ }^{43}$ publicó $60 \%$ de la presencia de hernia hiatal después de la cirugía y Felsenreich $^{44}$, confirma hernia hiatal de novo en el $45 \%$ y metaplasia de Barrett en 15\% de los pacientes, todos con reflujo sintomático; se sugiere que la hernia hiatal preexistente, la ERGE y el esófago de Barrett son contraindicaciones relativas para la GVT ${ }^{45-48}$. Varios autores han propuesto realizar una reparación de la hernia hiatal concomitante, algún tipo de fundoplicatura para prevenir los síntomas de reflujo de novo. Santoro ${ }^{45}$ publicó su técnica de hiatoplastia con una fundoplicatura anterior parcial, Nocca ${ }^{46}$ publicó recientemente sus resultados utilizando la GVT mas Nissen y nosotros publicamos nuestros resultados con este tipo de cirugía ${ }^{47}$. Gibson informó una tasa de disminución de ERGE de $41 \%$ preoperatoria a $7 \%$ después de la reparación combinada ${ }^{48}$. Soricelli y Daes presentaron resultados similares ${ }^{49,50}$.

Por el contrario, el estudio de Dakour ${ }^{51}$ comparó los pacientes sometidos a GVT con o sin la reparación concomitante de $\mathrm{HH}$ y los síntomas de ERGE no difirieron significativamente entre los dos grupos después de 1 y 2 años, con remisión en 21,3\% y $29,7 \%$ respectivamente, mientras que los síntomas de ERGE de novo se observaron en $41,4 \%$ y $46,2 \%$ respectivamente. Estos hallazgos significan que ambos procedimientos no protegieron a los pacientes de ERGE y respaldan los resultados informados por Samakar y Sannicola y otros autores que cuestionaron la eficacia de la reparación rutinaria de la hernia hiatal $^{52,53}$. Estamos de acuerdo con esta idea, porque

Tabla 2. Necesidad de tratamiento con inhibidores de la bomba de protones (IBP) (\% pacientes)

\begin{tabular}{|llll|}
\hline & Promedio & Rango & Referencias \\
Aumento de tratamiento (8 autores) & $46,5 \%$ & $3,3-61 \%$ & $(13,22,23,30-34)$ \\
Sin cambios en el tratamiento (1 autor) & $13 \%$ & & $(35)$ \\
Disminución del tratamiento (3 autores) & $55,5 \%$ & $40-71 \%$ & $(29,36,37)$ \\
Experiencia personal (pacientes bajo tratamiento) & $179 / 313(57,2 \%)$ & & \\
\hline
\end{tabular}


CIRUgíA AL díA

la reparación de la hernia hiatal, sola sin fundoplicatura, no mejora adecuadamente la presión del EEI (Tabla 3).

\section{Conclusiones}

Los estudios que evalúan la prevalencia de la enfermedad de reflujo gastroesofágico posoperatoria muestran que la GVT puede provocar síntomas de ERGE de novo o empeorar la ERGE preexistente $^{54,55}$. Si bien se necesitan más datos sobre la técnica y tiempo de seguimiento mayor para obtener conclusiones más definitivas, hasta ahora una importante cantidad de autores acepta que la GVT no es una buena operación para pacientes con ERGE y la mayoría de ellos prefieren bypass gástrico, que parece ser una mejor opción para estos pacientes.

\section{Responsabilidades éticas}

Protección de personas y animales. Los autores declaran que para esta investigación no se han realizado experimentos en seres humanos ni en animales.
Tabla 3. Esofagitis posoperatoria, esófago de Barrett y hernia hiatal después de GVT

\begin{tabular}{|lccc|}
\hline & $\begin{array}{c}\text { Experiencia } \\
\text { propia }\end{array}$ & \multicolumn{2}{c}{ Literatura } \\
Esango & (Referencia) \\
Esofagitis & $27,2 \%$ & $66-63$ & $(13,15,30-34)$ \\
Hernago de Barrett & $4,8 \%$ & $4,8-21$ & $(14,16)$ \\
$\quad$ Radiológica & & & \\
$\quad$ Endoscópica & $14,5 \%$ & $17,3-60,8 \%$ & $(13,43,44)$ \\
Conversion a bypass gástrico & $5,3 \%$ & $45 \%$ & $(43,44)$ \\
\hline
\end{tabular}

Confidencialidad de los datos. Los autores declaran que en este artículo no aparecen datos de pacientes.

Derecho a la privacidad y consentimiento informado. Los autores declaran que en este artículo no aparecen datos de pacientes.

Este estudio ha sido efectuado de acuerdo a las normas éticas de nuestro hospital y de acuerdo a la declaración de Helsinky.

Conflicto de Interés: sin conflictos de interés.

\section{Referencias}

1. Sammour T, Hill AG, Singh P, Ranasinghe A, Babor R, Rahman H. Laparoscopic sleeve gastrectomy as a single-stage bariatric procedure. Obes Surg. 2010;20:271-5.

2. Deitel M, Gagner M, Erickson AL, Crosby RD. Third International Summit: Current status of sleeve gastrectomy. Surg Obes Relat Dis. 2011;7:749-59. Obes Surg. 2006;16:1450-6.

3. Chiu S, Birch DW, Shi X, Sharma AM, Karmali S. Effect of sleeve gastrectomy on gastroesophageal reflux disease. Surg Obes Relat Dis. 2011;7:510-5.

4. Mahawar KK, Jennings N, Balupuri S, Small PK. Sleeve gastrectomy and gastrooesophageal reflux disease: a complex relationship. Obes Surg. 2013;23:987-91.

5. Gagner M, Deitel M, Kalberer TL, Erickson AL, Crosby RD. The Second International Consensus Summit for Sleeve Gastrectomy. Surg Obes Relat Dis. 2009;5:476-85.

6. Rosenthal RJ. International Sleeve Gastrectomy Expert Panel Consensus
Statement: best practice guidelines based on experience of 12,000 cases. Surg Obes Relat Dis. 2012;8:8-19.

7. Del Genio G, Tolone S, Limongelli P, Brusciano L, D'Alessandro A, Docimo G, et al. Sleeve Gastrectomy and Development of "De Novo" Gastroesophageal Reflux. Obes Surg. 2014;24:71-77.

8. Braghetto I, Lanzarini E, Korn O, Valladares H, Molina JC, Henriquez A. Manometric changes of the lower esophageal sphincter after sleeve gastrectomy in obese patients. Obes Surg. 2010;20:357-62.

9. Braghetto I, Csendes A, Korn O, Valladares H, González P, Henríquez A. Gastroesophageal reflux disease after sleeve gastrectomy. Surg Laparosc Endosc Percutan Tech. 2010;20:148-53.

10. Braghetto I. Davanzo C, Korn O, Csendes A, Valladares H, Herrera E, et al. Scintigraphic evaluation of gastric emptying in obese patients submitted to sleeve gastrectomy compared to normal subjects.Obes Surg. 2009;19:1515-21.

11. Melissas J, Braghetto I, Molina JC,
Silecchia G, Iossa A, Iannelli A, et al Gastroesophageal Reflux Disease and Sleeve Gastrectomy.Obes Surg. 2015;25:2430-5.

12. Himpens J, Dobbeleir J, Peeters G. Long term results of laparoscopic sleeve gastrectomy for obesity. Ann Surg. 2010;252:319-24.

13. Genco A, Soricelli E, Casella G, Maselli R, Castagneto-Gissey L, Di Lorenzo N, et al. Gastroesophageal reflux disease and Barrett's esophagus after laparoscopic sleeve gastrectomy: a possible, underestimated long-term complication. Surg Obes Relat Dis. 2017;13:568-74.

14. Tai CM, Huang CK, Lee YC, Chang CY, Lee CT, Lin JT. Increase in gastroesophageal reflux disease symptoms and erosive esophagitis 1 year after laparoscopic sleeve gastrectomy among obese adults. Surg Endosc. 2013;27:12606.

15. DuPree CE, Blair K, Steele SR. Laparoscopic sleeve gastrectomy in patients with preexisting gastroesophageal reflux disease: a national analysis. JAMA Surg. 2014;149:328-34. 
16. Oor JE, Roks DJ, Ünlü Ç, Hazebroek EJ. Laparoscopic sleeve gastrectomy and gastroesophageal reflux disease: a systematic review and meta-analysis. Am J Surg. 2016;211:250-67.

17. Altieri MS, Pryor AD. Gastroesophageal reflux disease after bariatric procedures. Surg Clin NA. 2015:95:579-91.

18. Tutuian R. Effects of bariatric surgery on gastroesophageal reflux. Curr Opin Gastroenterol. 2014;30:434-8.

19. Mion F, Tolone S, Garros A, Savarino E, Pelascini E, Robert M, et al. Highresolution Impedance Manometry after Sleeve Gastrectomy: Increased Intragastric Pressure and Reflux are Frequent Events. Obes Surg. 2016;26:2449-56.

20. Cottan D, Qureshi FG, Mattar SG, Laparoscopic sleeve gastrectomy as an initial weight-loss procedure for highrisk patients with morbid obesity. Surg. Endosc. 2006;20:859-63.

21. Yorke E, Sheppard C, Switzer NJ, Kim D, de Gara C, Karmali S, et al. Revision of sleeve gastrectomy to Roux-en-Y Gastric Bypass: A Canadian experience. Am J Surg. 2017;213:970-4.

22. Sheppard CE, Sadowski DC, de Gara CJ, Karmali S, Birch DW. Rate of reflux before and after laparoscopic sleeve gastrectomy for severe obesity. Obes Surg 2015;25:763-8.

23. Kowalewski PK, Olszewski R, Walędziak MS, Janik MR, Kwiatkowski A, GałązkaŚwiderek N. Long-Term Outcomes of laparoscopic Sleeve Gastrectomy-a Single-Center Retrospective Study. Obes Surg. 2018;28:130-4.

24. Mandeville Y, Van Looveren R, Vancoillie PJ, Verbeke X, Vandendriessche K, Vuylsteke P, et al. Moderating the Enthusiasm of Sleeve Gastrectomy: Up to Fifty Percent of Reflux Symptoms After Ten Years in a Consecutive Series of One Hundred Laparoscopic Sleeve Gastrectomies. Obes Surg. 2017;27:1797803.

25. Kasalický M. Bařinka A, Čierny M, Fried M, Gryga A, Holéczy P, et al. 10 years of sleeve gastrectomy in the Czech Republic in terms of the surgical procedure. Rozhl Chir. Winter 2016;95:425-31.

26. Chuffart E, Sodji M, Dalmay F, Iannelli A, Mathonnet M. Long-Term Results After Sleeve Gastrectomy for Gastroesophageal Reflux Disease: a Single-Center French
Study. Obes Surg. 2017;27:2890-7.

27. Albanopoulos K, Tsamis D, Natoudi M, Alevizos L, Zografos G, Leandros E. The impact of laparoscopic sleeve gastrectomy on weight loss and obesityassociated comorbidities: the results of 3 years of follow-up. Consecuencias clínicas posgastrectomía vertical tubular: Enfermedad por reflujo gastro-esofágico. Surg Endosc. 2016;30:699-705.

28. Goldenshluger M, Goldenshluger A, Keinan-Boker L, Cohen MJ, BenPorat T, Gerasi H, Amun M, et al. Postoperative Outcomes, Weight Loss Predictors, and Late Gastrointestinal Symptoms Following Laparoscopic Sleeve Gastrectomy. J Gastrointest Surg. 2017;21:2009-15.

29. Angrisani L, Santonicola A, Hasani A, Nosso G, Capaldo B, Iovino P. Five-year results of laparoscopic sleeve gastrectomy: effects on gastroesophageal reflux disease symptoms and co-morbidities. Surg Obes Relat Dis. 2016;12:960-8.

30. Catheline JM, Fysekidis M, Bachner I, Bihan H, Kassem A, Dbouk R, et al. Five year results of sleeve gastrectomy. J Visc Surg. 2013;150:307-12.

31. Carter PR, Leblanc A, Hausmann MG, Kleinpeter KP, de Barros SN, Jones SM, Association between gastroesophageal reflux disease and laparoscopic sleeve gastrectomy. Surg Obes Relat Dis. 2011;7:569-72.

32. Thereaux J, Lesuffleur T, Czernichow S, Basdevant A, Msika S, Nocca D, et al. Do sleeve gastrectomy and gastric bypass influence treatment with proton pump inhibitors 4 years after surgery? A nationwide cohort. Surg Obes Relat Dis. 2017;13:951-9.

33. Shi X, Karmali Sh, Sharma AM, Birsch DW. A review of laparoscopic sleeve gastrectomy for morbid obesity. Obes Surg. 2010;20:1171-7.

34. Bohdjalian A, Langer FB, ShakeriLeidenmühler S, Gfrerer L, Ludvik B, Zacherl J, et al. Sleeve gastrectomy as sole and definitive bariatric procedure: 5-year results for weight loss and ghrelin. Obes Surg. 2010;20:535-40.

35. Zhang N, Maffei A, Cerabona $\mathrm{T}$. Reduction in obesity-related comorbidities: is gastric bypass better than sleeve gastrectomy? Surg. Endosc. 201:27:1273-80.
36. Rebecchi F, Allaix ME, Giaccone C, Ugliono E, Scozzari G, Morino M. Gastroesophageal reflux disease and laparoscopic sleeve gastrectomy: a physiopathologic evaluation. Ann Surg. 2014;260:909-14.

37. Barry RG, Amiri FA, Gress TW, Nease DB, Canterbury TD. Laparoscopic vertical sleeve gastrectomy: A 5-year veterans affairs review. Medicine (Baltimore) 2017;96:e7508.

38. Mahawar KK, Jennings N, Balupuri S, Small PK. Sleeve gastrectomy and gastro-oesophageal reflux disease: a complex relationship Obes Surg. 2013;23:987-91.

39. Carabotti M, Silecchia G, Greco F, Leonetti F, Piretta L, Rengo M, et al. Impact of laparoscopic sleeve gastrectomy on upper gastrointestinal symptoms. Obes Surg. 2013;t;23:1551-7.

40. Chopra A, Chao E, Etkin Y. Merklinger L, Lieb J, Delany H. Laparoscopic sleeve gastrectomy for obesity: can it be considered a definitive procedure? Surg Endosc. 2012;26:831-7.

41. Weiner RA, Theodoridou S, Weiner S. Failure of laparoscopic sleeve gastrectomy-further procedure? Obes Facts. 2011;4 Suppl 1:42-6.

42. Braghetto I, Csendes A. Patients Having Bariatric Surgery: Surgical Options in Morbidly Obese Patients with Barrett's Esophagus. Obes Surg. 2016;26:1622-6.

43. Rengo M, Bellini D, Iorio O, De Cecco CN, Rizzello M, Cavallaro G, et al. Role of preoperative imaging with multidetector computed tomography in the management of patients with gastroesophageal reflux disease symptoms after laparoscopic sleeve gastrectomy. Obes Surg. 2013;23:1981-6.

44. Felsenreich DM, Kefurt R, Schermann M, Beckerhinn P, Kristo I, Krebs M, et al. Reflux, Sleeve Dilation, and Barrett's esophagus after Laparoscopic Sleeve Gastrectomy: Long-Term Follow-Up. Obes Surg. 2017;27:3092-101.

45. Santoro S, Lacombe A, Aquino CG. Malzoni CE. Sleeve gastrectomy with antireflux procedure. Einstein( Sao Paulo) 2014:12:287-94.

46. Nocca D, Skalli EM. Nissen- Sleeve (N-Sleeve) operation: preliminary results of a pilot study. SOARD 2016:1-6.

47. Lasnibat JP, Braghetto I. Gutierrez L, 
CIRUgíA AL díA

Sanchez F. Sleeve gastrectomy and fundoplication as a single procedure in patients with obesity and gastroesophageal reflux. Arq Bras Cir Dig. 2017;30:216-21.

48. Gibson SC, Le Page PA, Taylor CJ. Laparoscopic sleeve gastrectomy: review of 500 cases in single surgeon Australian practice. ANZ J Surg. 2015:85:673-7.

49. Soricelli E, Iossa A, Casella G, Abbatini F, Calì B, Basso N. Sleeve gastrectomy and crural repair in obese patients with gastroesophageal reflux disease and/ or hiatal hernia. Surg Obes Relat Dis. 2013;9:356-61.

50. Daes, Jimenez ME, Said N, Dennis R. Improvement of gastroesophageal reflux symptoms after standardized laparoscopic sleeve gastrectomy. Obes Surg.

2014;24:536-40

51. Dakour Aridi H, Asali M, Fouani T, Alami RS, Safadi BY. Gastroesophageal Reflux Disease After Laparoscopic Sleeve Gastrectomy with Concomitant Hiatal Hernia Repair: an Unresolved Question. Obes Surg. 2017;27:2898-04.

52. Samakar K, McKenzie TJ, Tavakkoli A, Vernon AH, Robinson MK, Shikora SA. The Effect of laparoscopic Sleeve Gastrectomy with Concomitant Hiatal Hernia Repair on Gastroesophageal Reflux Disease in the Morbidly Obese. Obes Surg. 2016;26:61-6.
53. Santonicola A, Angrisani L, Cutolo P, Formisano G, Iovino P. The effect of laparoscopic sleeve gastrectomy with or without hiatal hernia repair on gastroesophageal reflux disease in obese patients. Surg Obes Relat Dis. 2014:10;250-5.

54. Himpens J. Comment on: Gastroesophageal reflux in laparoscopic sleeve gastrectomy: hiatal findings and their management influence outcome. Surg Obes Relat Dis. 2015;11:537-8.

55. Nadaleto BF, Herbella FA, Patti MG. Gastroesophageal reflux disease in the obese: Pathophysiology and treatment. Surgery 2016;159:475-86. 